\title{
Resting State Functional Connectivity and Thrombolysis Mediated Reperfusion in Acute Ischemic Stroke: A Pilot Study
}

\author{
Priyantha Herath, Taylor Hanayik, Kaitlin Krebs, Lauren Dennis, Christopher Rorden, Julius Fridriksson and Souvik Sen*
}

Department of Neurology, McCausland Center for Brain Imaging, University of South Carolina School of Medicine, Columbia, South Carolina, USA

\begin{abstract}
Introduction: Intravenous Tissue Plasminogen Activator (rTPA) is used to treat acute ischemic stroke (AIS). Early recanalization from this leads to better stroke outcomes, but the exact mechanism remains unknown. To clarify this, we correlated tissue perfusion and functional outcomes in acute stroke patients (AIS) who received rTPA to resting state default mode network (DMN) and task-positive (TPN) activities measured with fMRI. Following NIH stroke scale (NIHSS) assessments, patients underwent magnetic resonance imaging (MRI) scans during rTPA infusion (baseline), six hours post stroke and at $30 \mathrm{~d}$ follow up visit.

Results: Paired t-tests revealed that NIHSS at 6 hrs post stroke and at 30-days follow up significantly better compared to baseline, indicating improved functional outcomes. Changes in NIHSS were associated by significant changes in resting connectivity in TPN and DMN. In the TPN, both the undamaged Frontal Eye Field (FEF) and the undamaged intraparietal sulcus (IPS) node at the $6 \mathrm{hrs}$ time point improved in connectivity with other TPN nodes compared to baseline. 30 days follow up resting connectivity of the DMN on the medial-prefrontal (MPF) node, and undamaged lateral parietal (LP) node, along with the damaged medial-temporalnode of TPN showed more robust correlations from baseline to 30 days follow up. The damaged IPS connectivity was the only measure that significantly correlated with NIHSS at the $6 \mathrm{hrs}$ time point. No correlations with NIHSS were found at baseline or the 30 days time points with resting state or perfusion data.

Conclusion: In this pilot study, we found that patients who received rTPA showed changes in resting state networks and functional outcomes over time. These findings point to an intriguing possibility, that the improvement of resting state networks may reflect improved efficiency of brain activity that is potentially related to functional outcomes in AIS patients who receive rTPA. As such, the improved functional connectivity measured with rSBOLD fMRI should be further explored as a potential predictive biomarker for rTPA response. Larger studies are needed to verify these findings.
\end{abstract}

Keywords: Resting state functional MRI; Cerebral perfusion; Thrombolysis; NIHSS; Outcome measures; Prediction; Stroke

\section{Introduction}

At present, the only FDA approved therapy for acute ischemic strokes is intravenous tissue plasminogen activator (rTPA) [1]. The use of IV rTPA is associated with improved outcomes for a broad spectrum of patients who can be treated within 3 hours or 3-4.5 hours of stroke onset $[2,3]$. When a cerebral artery is occluded, as occurs in ischemic stroke, regional cerebral blood flow (rCBF) drops precipitously within a central core region of brain supplied by that artery, but that core is surrounded by regions (stroke penumbra) of progressively increasing $\mathrm{rCBF}$ where perfusion is maintained by collateral circulation. During acute ischemic stroke, the level of perfusion determines the eventual core lesion size, the resulting stroke deficits and functional outcome [4]. Prior studies have documented that patients with large vessel occlusions have more severe stroke volumes and worse prognosis than those without [5-8], and are likely to be poor responders to rTPA $[2,9,10]$. If this is so, the long term outcomes are also likely to be less favorable. A recent thrombolytic study suggested that early recanalization may be associated with better functional outcomes after an ischemic stroke [11], but concerns have been raised that conventional measures of improvement based on NIHSS alone after rTPA may not be reliable predictors for future outcomes after an ischemic stroke [12].

It is well established in neuroscience that the role of widely distributed neural networks is important for the control of neurological functions [13-15]. A network perspective, which is now thought to be a more biologically plausible model for how the brain works [16-18], suggests that physiological effects of brain injury are best assessed over entire networks rather than just locally at the site of structural damage $[1,19,20]$. However, it is entirely unknown what patterns of interaction within brain neuronal networks are most closely associated with behavioral deficits after ischemic tissue injury.

Resting state functional connectivity (FC) magnetic resonance imaging (MRI) (rsf MRI) [21] measures, within a subject, the temporal correlation of the blood oxygenation level dependent (BOLD) signal across regions without any imposed task, providing a measure of temporal coherence of activity between brain regions. Recent studies indicate that such functional networks identified with the use of resting FC measurements strongly overlap with networks activated by task performance [22], and that spontaneous activity correlates with trial-to-trial fluctuations in task-evoked responses [23] and behavior $[22,24]$. The resting FC methodology circumvents the need for taskoriented behavioral tests that could be extremely cumbersome in the setting of acute ischemic stroke. The purpose of this pilot study was

*Corresponding author: Sen S, Department of Neurology, University of South Carolina School of Medicine, 8 Medical Park, Suite 420, Columbia, South Carolina, USA, Tel: 803-545-6050/6073; Fax: 803-545-6051; E-mail: Souvik.Sen@uscmed.sc.edu

Received January 26, 2015; Accepted March 27, 2015; Published March 29, 2015

Citation: Herath P, Hanayik T, Krebs K, Dennis L, Rorden C, et al. (2015) Resting State Functional Connectivity and Thrombolysis Mediated Reperfusion in Acute Ischemic Stroke: A Pilot Study. J Neurol Disord 3: 228. doi: 10.4172/23296895.1000221

Copyright: ( 2015 Herath $\mathrm{P}$, et al. This is an open-access article distributed under the terms of the Creative Commons Attribution License, which permits unrestricted use, distribution, and reproduction in any medium, provided the original author and source are credited. 
to correlate DMN and TPN resting state network activity with tissue perfusion and relevant functional outcomes, in stroke patients who received rTPA.

\section{Materials and Methods}

Acute ischemic stroke patients who qualified for rTPA protocol were consented for an institutional review board approved sequential MRI protocol. An MRI safety screening questionnaire was completed to ensure patients were eligible to have an MRI. The MRI protocol included three sets of images obtained at baseline (during rTPA infusion), six-hours and thirty-days post symptom onset. Scans were performed using a 3T Siemens Trio scanner, (12-channel head coil) located adjacent to the Emergency Room. These patients underwent an abbreviated MRI scan to verify the presence of brain ischemia (brain attack diffusion weighted MRI) as a part of our routine standard of care. The resting state fMRI was therefore the additional MR acquisition that was done experimentally. In an effort to avoid delay of treatment with rTPA, consent was obtained either before rTPA was administered or shortly afterwards; however, the baseline MRI scan was initiated within 30 minutes of initiating rTPA treatment in the patient. The patients who were found not to have ischemia on the baseline MRI were excluded. Processing for MR images was identical for the baseline and six-hour ( $6 \mathrm{hrs})$ post rTPA time points.

\section{MRI diffusion and perfusion imaging}

The imaging sequences include an initial diffusion-weighted sequence: EPI, repetition time/echo time (TR/TE), 2900/78 ms; $b=0$, $500,1000 \mathrm{~s} / \mathrm{mm} \mathrm{2} 3$ axis encoding; matrix, $128 \times 128$ pixels. All images were acquired in transverse plane with identical position for each patient, sectional thickness $(5 \mathrm{~mm})$, intersection gap ( $1 \mathrm{~mm})$, and FOV $(230 \mathrm{~mm})$. This was followed by contrast (a standard dose of IV Gadolinium; $100 \mathrm{~mL}$ ) enhanced perfusion images obtained at baseline and six-hours from symptom onset using the following parameters: Axial echo-planar imaging (EPI) scan, TR=1580 ms, TE $=43 \mathrm{~ms}$, flip angle $=90^{\circ}, \mathrm{FoV}=230 \mathrm{~mm}$, matrix size $=128 \times 128$, with 18 slices each $5 \mathrm{~mm}$ thick. Following data collection, we chose the auto-generated Siemens percentage of baseline at peak (PBP) and Time to Peak (TTP) images as our measures of perfusion and used these modalities for our SPM12 analysis pipeline that involved normalization, and parcellation using the Johns Hopkins University (JHU) atlas of cortical brain regions [25]. The PBP image shows the signal change of the bolus peak relative to the baseline. Brighter voxels in this modality indicate less contrast agent arrived. On the other hand, TTP images show time from first appearance of bolus in the artery to peak signal change observed in tissue. Since the auto-generated PBP and TTP maps are not computed for all voxels (e.g. signal drop out in large sulci and near the ventricles) neither voxel-wise analyses nor conventional smoothing was appropriate for these data. Therefore, normalization used nearest neighbor approximations and mean signal within each region of interest in the atlas based only on voxels where a signal was present. After normalization to a mirror-symmetric template included in the clinical toolbox [26], the images from individuals with left hemisphere injury were left-right flipped so that all stroke damage appeared in the right hemisphere for each individual (regardless of whether the patient had a left or a right hemisphere stroke). Left-right flipping the images allowed for greater statistical power during later inferential statistics.

\section{Resting state functional MRI}

Resting state BOLD (rsBOLD) images were obtained at baseline, 6-hours, and 30 days from symptom onset using the following param- eters: T2*-weighted GRE-EPI sequence, with interleaved acquisition of slices, a $256 \times 256 \mathrm{~mm}$ field of view (FoV), $80^{\circ}$ flip angle, TR=2000 $\mathrm{ms}, \mathrm{TE}=32 \mathrm{~ms}$, and 34 interleaved slices for 150 volumes. Each slice was $4 \mathrm{~mm}$ thick with a $50 \%$ gap resulting in $6 \mathrm{~mm}$ between voxel centers. An anatomical image was also collected for each participant with the following parameters: T1 MPRAGE sequence, with ascending acquisition of slices, a $256 \times 256 \mathrm{~mm}$ field of view (FoV), $15^{\circ}$ flip angle, $\mathrm{TR}=1520 \mathrm{~ms}, \mathrm{TE}=3.69 \mathrm{~ms}$, and 144 slices. Each slice was $1 \mathrm{~mm}$ thick with a $50 \%$ gap resulting in $1.5 \mathrm{~mm}$ between voxel centers. Resting state images were processed in SPM12 using a standard pipeline of slice time correction, motion correction, coregistration, normalization, frequency filtering with a 0.01 to $0.1 \mathrm{~Hz}$ band-pass [27] spatial smoothing ( $8 \mathrm{~mm}$ FWHM), and parcellation into TPN and DMN nodes based on the work of Fox et al. [22] Standard Pearson correlations were calculated to explore the relationship between and within these networks.

\section{MRI post-processing}

Each participant's perfusion scan was parcellated into cortical regions using the JHU atlas. Using these parcellated images, we then averaged every region in each hemisphere resulting in a hemispheric average perfusion value based on these cortical locations. Generating hemispheric averages for perfusion was necessary due to slightly varying plane differences in the FoV for the clinical perfusion scans, which resulted in no datas for some regions in the parcellated images. In other words, the FoV was kept at a constant size across participants.

\section{Clinical assessments}

To measure clinical outcomes that may be associated with functional connectivity in the brain, the patients were assessed using clinical scales at baseline at the time of arrival at the ED prior to administration of rTPA, after rTPA has been given, and at 30 days. Prior to the post stroke day $30 \mathrm{MRI}$, patients underwent a clinical evaluation by one of the study investigators. This evaluation included the interval history and neurological examination (including NIH stroke scale -NIHSS). The following information was obtained and recorded: development of recurrent signs or symptoms consistent with a recurrent stroke, NIHSS, and disability scores.

\section{Statistical analysis}

Standard parametric statistics were computed for images in this data set. The TPN and DMN individual node correlations were averaged across patients and fed to subsequent analyses that included repeated measures t-tests (baseline during rTPA administration and sixhours from stroke symptom onset), and Pearson correlations using Fisher $\mathrm{z}$ transformed correlation values from rsf MRI data. We aimed to investigate changes that are likely to be subsequent to rTPA, and the effects on patient outcome as measured by the NIHSS. The perfusion images were averaged across patients by hemisphere and used in the same analysis procedures. We chose not to use the analysis of variance (ANOVA) to analyze the differences between groups as the data sets were dependent of one another.

\section{Results}

Fourteen patients (mean age $\pm \mathrm{SD}=63 \pm 14 ; 7$ male, $50 \%$ white, $43 \%$ black and $7 \%$ other; 3 with a left MCA stroke, 8 with a right MCA stroke, 3 other) who qualified for rTPA protocol consented and completed the MRI scan at baseline and 6 hours post stroke onset. rTPA was administered using a MR safe IV infusion pump per NINDS protocol with an average door to needle time of 55 minutes (range 25-110 minute) with door to needle time $<60 \mathrm{~min}$ in 11 of the $14 \mathrm{sub}$ - 
Citation: Herath P, Hanayik T, Krebs K, Dennis L, Rorden C, et al. (2015) Resting State Functional Connectivity and Thrombolysis Mediated Reperfusion in Acute Ischemic Stroke: A Pilot Study. J Neurol Disord 3: 228. doi: 10.4172/2329-6895.1000221

Page 3 of 5

jects. Three of these patients were found not to have ischemia and one patient had an incomplete series of scans. These four patients were excluded resulting in 10 sets of patient data for analysis. Of these, only six patients had valid follow up data at 30 days, as three expired prior to 30-day visit and one was unable to return for follow-up MRI scan due to stroke related disability. NIHSS scores at $6 \mathrm{hrs}$ post stroke $(\mathrm{M}=7.5, \mathrm{SD}=5.3) \mathrm{t}(9)=3.2, \mathrm{p}<0.05$, Hedges [27] $\mathrm{g}=0.87$ and at 30 -days follow up $(M=4.7, S D=6) t(5)=3.55, p<0.05, g=1$ were significantly improved from the NIHSS scores at baseline $(\mathrm{M}=12, \mathrm{SD}=5.7)$, indicating that functional outcome as measured by the NIHSS is improved across participants (Figure 1). The TPN node IPS on the damaged side was the only locus that was directly and significantly correlated with NIHSS score at the $6 \mathrm{hrs}$ time point ( $\mathrm{r}=-0.82$, $\mathrm{q}$ (FDR corrected) $<0.05$ ) (Figure 2). No correlations with NIHSS were found at baseline or the 30-day time points. In the meantime, the changes in NIHSS corresponded to a reciprocal and significant change in resting connectivity in TPN and DMN as measured by MRI (Figure 3). Both the left Frontal Eye Field (FEF) node (baseline: $\mathrm{M}=-0.05, \mathrm{SD}=0.12,6 \mathrm{hrs}$ : $\mathrm{M}=0.08, \mathrm{SD}=0.11) \mathrm{t}(9)=-4.9, \mathrm{p}<0.001, \mathrm{~g}=1.2$ and the left intraparietal sulcus (IPS) node (baseline: $\mathrm{M}=0.09, \mathrm{SD}=0.1,6 \mathrm{hrs:} \mathrm{M}=0.19, \mathrm{SD}=0.14$ ) $t(9)=-2.7, p<0.05, g=0.86$ showed improved in connectivity with other task positive nodes at the $6 \mathrm{hrs}$ time point compared to baseline. Although 30-day follow up resting connectivity data were only available in 6 participants, DMN network connectivity on the medial-prefronal (MPF) node (baseline: $\mathrm{M}=0.15, \mathrm{SD}=0.21,30$ day: $\mathrm{M}=0.42, \mathrm{SD}=0.2$ ) $\mathrm{t}(5)=-2.9, \mathrm{p}<0.05, \mathrm{~g}=1.2$, and left lateral parietal (LP) node (baseline: $\mathrm{M}=0.25, \mathrm{SD}=0.31,30$ day: $\mathrm{M}=0.5, \mathrm{SD}=0.26) \mathrm{t}(5)=-2.7, \mathrm{p}<0.05, \mathrm{~g}=0.82$, along with the TPN right medial-temporal node (baseline: $\mathrm{M}=0.01$, $\mathrm{SD}=0.13,30$ day: $\mathrm{M}=0.14, \mathrm{SD}=0.09) \mathrm{t}(5)=-2.8, \mathrm{p}<0.05, \mathrm{~g}=1$ showed improvement in those 6 participants from baseline to 30 -days follow up (Table 1).

\section{Discussion}

Our study revealed several intriguing preliminary findings. Foremost, it establishes the feasibility of conducting resting functional connectivity in conjunction with acute stroke thrombolysis without adversely influencing the door to needle time or clinical outcome. This alone is a novel and important methodological issue that is worth noting, given the ubiquitous availability and ease of use of resting state fMRI. In our small sample, we note that the IPS functional connectivity on the damaged hemisphere was robustly correlated with

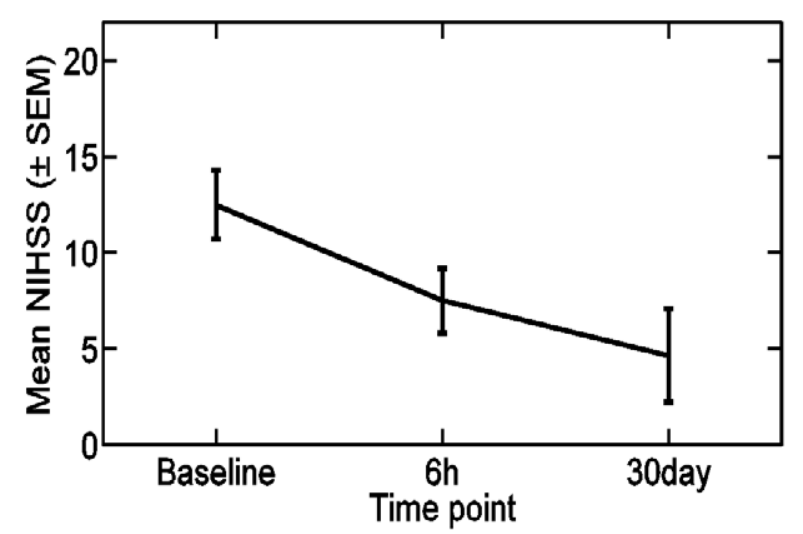

Figure 1: Improvement of NIHSS scores over time from base line to $6 \mathrm{~h}$ post stroke $(M=7.5, S D=5.3) t(9)=3.2, p<0.05$, Hedges [26] $g=0.87$ and at 30 -days follow up $(M=4.7, S D=6) t(5)=3.55, \quad p<0.05, g=1$ were significantly improved from the NIHSS scores at baseline $(M=12, S D=5.7)$, indicating how the functional outcomes improved.

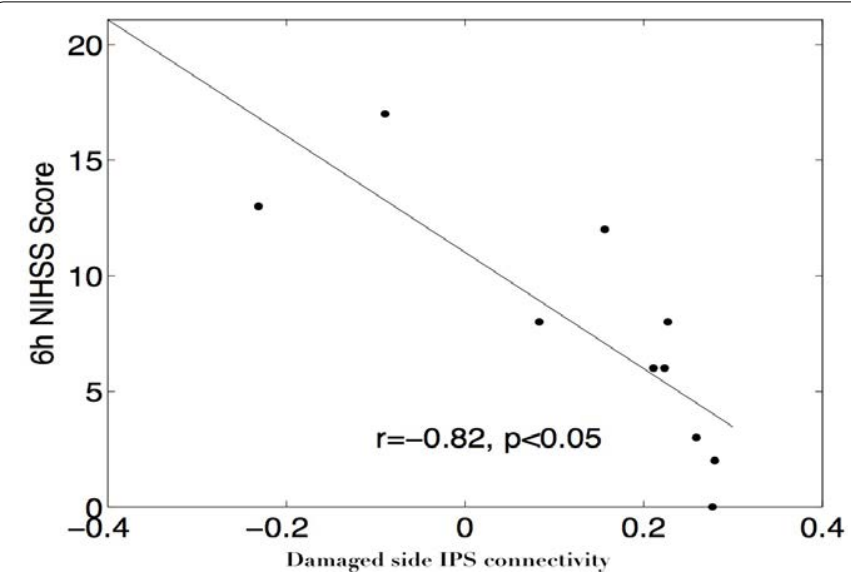

Figure 2: This graph shows the correlation between NIHSS score at the $6 \mathrm{~h}$ time point and the TPN node IPS on the damaged side $(r=-0.82$, q (FDR corrected) <0.05)
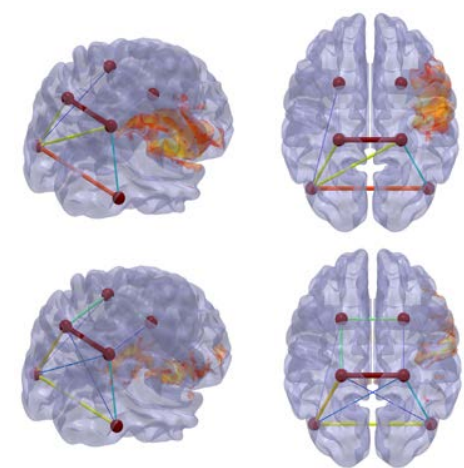

Figure 3: The top panels show rsBOLD data and PBP perfusion data at baseline. The bottom panel illustrates the rSBOLD and PBP data at 6 hours post TPA infusion. The PBP measure indicates areas of reduced perfusion and "hotter" colors indicate a greater reduction in signal (i.e. lower perfusion). PBP data have been smoothed using a $6 \mathrm{~mm}$ FWHM Gaussian kernel in SPM12 and thresholded to maximize the reduction of noise due to signal dropout around the ventricles and skull. Resting state connectivity data are represented by nodes and lines connecting the nodes together. The temperature and thickness of the lines indicate the strength of the connections, with warmer, and thicker lines indicating more correlational connectivity.

\begin{tabular}{|c|c|c|c|c|c|c|}
\hline \multirow{3}{*}{ Baseline } & \multicolumn{2}{|c|}{ NIHSS } & \multicolumn{2}{|c|}{$\begin{array}{l}\text { Left Frontal Eye Field } \\
\text { (FEF) Node (TPN) }\end{array}$} & \multicolumn{2}{|c|}{$\begin{array}{l}\text { Left Intraparietal } \\
\text { Sulcus (IPS) Node } \\
\text { (TPN) }\end{array}$} \\
\hline & $M=12$ & \multirow{2}{*}{ vs. Baseline } & $M=-0.05$ & \multirow{2}{*}{ vs. Baseline } & $M=0.09$ & $\begin{array}{c}\text { vs. } \\
\text { Baseline }\end{array}$ \\
\hline & $\mathrm{SD}=5.7$ & & $S D=0.12$ & & $S D=0.1$ & \\
\hline $6 \mathrm{hr}$ & $\begin{array}{c}M=7.5 \\
S D=5.3\end{array}$ & $\begin{array}{l}t(9)=3.2 \\
p<0.05^{*}\end{array}$ & $\begin{array}{l}M=0.08 \\
S D=0.11\end{array}$ & $\begin{array}{l}t(9)=-4.9 \\
p<0.001^{*}\end{array}$ & $\begin{array}{c}M=0.19 \\
S D=0.14\end{array}$ & $\begin{array}{c}t(9)=-2.7 \\
p<0.05^{*}\end{array}$ \\
\hline \multirow{3}{*}{30 day } & $M=4.7$ & $t(5)=3.55$ & & & & \\
\hline & $\mathrm{SD}=6$ & $\mathrm{p}<0.05^{\star}$ & & & & \\
\hline & \multicolumn{2}{|c|}{$\begin{array}{l}\text { Medial-Prefrontal } \\
\text { (MPF) Node(DMN) }\end{array}$} & \multicolumn{2}{|c|}{$\begin{array}{l}\text { Left Lateral Parietal } \\
\text { (LP) Node(DMN) }\end{array}$} & \multicolumn{2}{|c|}{$\begin{array}{l}\text { Right Medial- } \\
\text { Temporal Node } \\
\text { (TPN) }\end{array}$} \\
\hline \multirow{2}{*}{ Baseline } & $M=0.15$ & vs. Baseline & $M=0.25$ & vs. Baseline & $M=0.01$ & $\begin{array}{c}\text { vs. } \\
\text { Baseline }\end{array}$ \\
\hline & $S D=0.21$ & & $S D=0.31$ & & $S D=0.13$ & \\
\hline \multirow{2}{*}{30 day } & $M=0.42$ & $t(5)=-2.9$ & $M=0.5$ & $t(5)=-2.7$ & $M=0.14$ & $t(5)=-2.8$ \\
\hline & $S D=0.2$ & $p<0.05^{*}$ & $S D=0.26$ & $\mathrm{p}<0.05^{*}$ & $S D=0.09$ & $p<0.05^{*}$ \\
\hline
\end{tabular}

Table 1: Significant statistical results of behavioral and neuroimaging data at baseline, 6 hours, and 30 days. 
improvement in neurological status as evidenced by the decrement in NIHSS. These findings point to the exciting possibility that resting state functional MRI data may compliment previously reported ADC based findings, which had been used to provide a reliable risk assessment for brain injury; however, due to uncertainties of reperfusion, these ADC maps could not predict future outcome in animals [28]. As such, we show here that rsfMRI might be a useful, and perhaps additive biomarker used to determine functional prognosis in AIS patients. The findings are in agreement with a recent human study that demonstrated that rsfMRI scans have the potential to estimate brain tissue viability after acute ischemia [29]. To our knowledge our study here is the first attempt at feasibility of resting functional MRI along with perfusion imaging to predict outcome after acute stroke thrombolysis.

A recent non-acute stroke study demonstrated the utility of concurrent BOLD and rCBF signals from ASL perfusion MRI for assessing resting brain function [30]. That particular study raised the possible utility of correlating perfusion imaging and resting state functional connectivity. Here, we were not able to replicate this finding, quite likely due to our small statistical sample size, based on our observation that there was no significant difference between baseline and 6 hrs mean hemispheric PBP (which measures decreased perfusion). The lack of statistical difference in our perfusion values could be attributed to our averaging method, which may have masked any important differences that we were interested in. Unfortunately, the hemispheric averaging was necessary for perfusion data due to large signal dropout near the ventricles and other areas. Still, our finding of an improvement on the resting networks after rTPA indicates that resting state connectivity might serve as a MRI surrogate for functional outcomes after stroke. The fact that these networks were not negatively correlated as in healthy brain tissue might indicate that the observed changes in the networks are those that appear in damaged network systems, rather than in healthy networks. Moreover, the noted DMN improved connectivity, but not the TPN from 6 hours post stroke to 30 -days follow up, with improved 30 -day functional outcomes measured by NIHSS leads to speculation of how differential networks may takeover different stages of functional recovery after stroke. All of these findings may be confounded by small sample size, and therefore our intent is merely to report a potential methodological approach and to suggest long-term follow-up with MRI imaging that would help understand the physiology of these very intriguing findings.

The findings we report here lead to several important considerations. First, with statistical tests that are considered appropriate for small samples we have found significant correlations between functional connectivity and clinical outcomes following thrombolysis for AIS, this may lead to the consideration of the possibility that rsfMRI may provide an additional, adequately robust predictive method when the application of this technique for acute ischemic stroke settings is developed. Because main mechanisms underlying functional recovery after the acute phase of an ischemic stroke are quite likely determined by functional reorganization of distributed brain networks, non-invasive functional connectivity mapping is a useful tool, which is widely available to clinicians who need to understand the prognostic data for their patients. It is also intriguing to speculate whether the improvement of resting state connectivity over time is perhaps a reflection of recovery achieved by plastic changes in response to disconnection caused by remote degeneration affects in distant brain regions because of losses in white matter (WM) integrity. As we mentioned before, the human brain works by way of networked activity [31], such a scenario can speculatively explain the initial functional deficits that are grossly captured by NIHSS and the subsequent improvement of NIHSS over time, thus suggesting that perhaps rsfMRI is possibly an additive, and perhaps a more objective measure of functional recovery for acute stroke.

Our findings also lead to a number of pathophysiological questions that must be investigated in the future. For example, given impaired cerebral auto regulation that occurs during the acute stage of an ischemic stroke, cerebral blood flow, perfusion, and subsequently the BOLD signal characteristics are all likely to be different from normal, healthy brain tissue. While thrombolytics are known to lead to improved tissue perfusion in the penumbra of a stroke, our study did not reveal an association between perfusion and NIHSS, even as functional connectivity improved. So, it is worthy speculating whether the clinical improvements occur not merely due to perfusion but perhaps due to improved and plastic nature of the network connectivity. In the meantime, the correlations we have noted reaffirm the relative robustness of rsFMRI as a potential future predictive modality in AIS patients.

There are methodological limitations to the preliminary research we report here. The results of the small pilot study must be interpreted with caution. For example, even though we examined rsfMRI data with a view to seek changes that are likely to be subsequent to TPA, exact elucidation of such changes requires a control group. Overall, our findings and methods should be carefully utilized in designing a larger definitive study with a control arm. In addition to confirming the above finding, a larger case-control study will allow the investigation of individual networks such as visual, auditory and sensorimotor networks, in addition to the default-mode network $[32,33]$.

\section{Conclusion}

In this preliminary feasibility study we found that rTPA is associated with changes in MRI based resting state networks and associated functional outcome. Correlations were found between functional connectivity and NIHSS but not with perfusion. These correlations point to the possibility that the improvement of resting state networks means improved efficiency of brain activity indicated by functional outcome and may be a potential predictive MRI biomarker for rTPA response. Larger studies with control arms are needed to verify this finding, and further ascertain the potential role of network changes that may be predictive of stroke recovery.

\section{Acknowledgement}

This research work has been supported by NIH grant: R01NS062754

\section{References}

1. Adams HP Jr, del Zoppo G, Alberts MJ, Bhatt DL, Brass L, et al. (2007) Guidelines for the early management of adults with ischemic stroke: a guideline from the American Heart Association/American Stroke Association Stroke Council, Clinical Cardiology Council, Cardiovascular Radiology and Intervention Council, and the Atherosclerotic Peripheral Vascular Disease and Quality of Care Outcomes in Research Interdisciplinary Working Groups: the American Academy of Neurology affirms the value of this guideline as an educational tool for neurologists. Stroke 38: 1655-1711.

2. The National Institute of Neurological Disorders and Stroke rt-PA Stroke Study Group (1995) Tissue plasminogen activator for acute ischemic stroke. N Engl J Med 333: 1581-1587.

3. Hacke W, Kaste M, Bluhmki E, Brozman M, Dávalos A, et al. (2008) Thrombolysis with alteplase 3 to 4.5 hours after acute ischemic stroke. N Engl J Med 359: 1317-1329. 
Citation: Herath P, Hanayik T, Krebs K, Dennis L, Rorden C, et al. (2015) Resting State Functional Connectivity and Thrombolysis Mediated Reperfusion in Acute Ischemic Stroke: A Pilot Study. J Neurol Disord 3: 228. doi: 10.4172/2329-6895.1000221

4. Sen S (2009) Magnetic Resonance Imaging in Acute Stroke: eMedicine Neurology. Medscape

5. Bastianello S, Pierallini A, Colonnese C, Brughitta G, Angeloni U, et al. (1991) Hyperdense middle cerebral artery CT sign. Comparison with angiography in the acute phase of ischemic supratentorial infarction.Neuroradiology 33: 207211.

6. Launes J, Ketonen L (1987) Dense middle cerebral artery sign: an indicator of poor outcome in middle cerebral artery area infarction. J Neurol Neurosurg Psychiatry 50: 1550-1552.

7. Moulin T, Cattin F, Crépin-Leblond T, Tatu L, Chavot D, et al. (1996) Early CT signs in acute middle cerebral artery infarction: predictive value for subsequent infarct locations and outcome. Neurology,. 47: 366-375.

8. Zorzon M, Masè G, Pozzi-Mucelli F, Biasutti E, Antonutti L, et al. (1993) Increased density in the middle cerebral artery by nonenhanced computed tomography. Prognostic value in acute cerebral infarction. Eur Neurol, 33: 256 259.

9. Manelfe C, Larrue V, von Kummer R, Bozzao L, Ringleb P, et al. (1999) Association of hyperdense middle cerebral artery sign with clinical outcome in patients treated with tissue plasminogen activator. Stroke 30: 769-772.

10. Tomsick T, Brott T, Barsan W, Broderick J, Haley EC, et al. (1996) Prognostic value of the hyperdense middle cerebral artery sign and stroke scale score before ultraearly thrombolytic therapy. AJNR Am J Neuroradiol 17: 79-85.

11. Olivot JM, Mlynash M, Thijs VN, Kemp S, Lansberg MG, et al. (2008) Relationships between infarct growth, clinical outcome, and early recanalization in diffusion and perfusion imaging for understanding stroke evolution (DEFUSE). Stroke 39: 2257-2263.

12. Johnston KC, Connors AF Jr, Wagner DP, Knaus WA, Wang X, et al. (2000) A predictive risk model for outcomes of ischemic stroke. Stroke 31: 448-455.

13. Felleman DJ, Van Essen DC (1991) Distributed hierarchical processing in the primate cerebral cortex. Cereb Cortex 1: 1-47.

14. Goldman-Rakic PS (1988) Topography of cognition: parallel distributed networks in primate association cortex. Annu Rev Neurosci, 11: 137-156.

15. Posner MI, Petersen SE, Fox PT, Raichle ME (1988) Localization of cognitive operations in the human brain. Science 240: 1627-1631.

16. Hebb DO (1949) The Organization of Behavior: A Neuropsychological Theory (1st edn), Taylor \& Francis,New Jersey, USA.

17. Shepherd GM (2003) The synaptic organization of the brain (5th edn), Oxford University Press, USA.

18. Sporns O (2003) Network Analysis, Complexity, and Brain Function. Complexity 8: $56-60$.
19. Honey CJ, Sporns O (2008) Dynamical consequences of lesions in cortical networks. Hum Brain Mapp 29: 802-809.

20. Von Monakow C (1911) Localization of brain functions. J Psychol Neurol 17: 185-200.

21. Biswal B, Yetkin FZ, Haughton VM, Hyde JS (1995) Functional connectivity in the motor cortex of resting human brain using echo-planar MRI. Magn Reson Med 34: 537-541.

22. Fox MD, Raichle ME (2007) Spontaneous fluctuations in brain activity observed with functional magnetic resonance imaging. Nat Rev Neurosci 8: 700-711.

23. Fox M D, Snyder AZ, Zacks JM, Raichle ME, et al. (2006) Coherent spontaneous activity accounts for trial-to-trial variability in human evoked brain responses. Nat Neurosci 9: 23-25.

24. Hampson M, Driesen NR, Skudlarski P, Gore JC, Constable RT (2006) Brain connectivity related to working memory performance. J Neurosci 26: 1333813343.

25. Andreia VF, Kenichi O, Susumu M (2010) Study of white matter anatomy and $3 \mathrm{D}$ tract reconstruction by diffusion tensor imaging. International Journal of Imaging Systems and Technology 20: 51-56.

26. Rorden C, Bonilha L, Fridriksson J, Bender B, Karnath HO, et al. (2012) Agespecific CT and MRI templates for spatial normalization. Neuroimage 61: 957 965 .

27. Hallquist MK, Hwang, Luna B (2013) The nuisance of nuisance regression spectral misspecification in a common approach to resting-state fMR preprocessing reintroduces noise and obscures functional connectivity. Neuroimage 82: 208-225.

28. Pillekamp F, Grüne M, Brinker G, Franke C, Uhlenküken U, et al. (2001) Magnetic resonance prediction of outcome after thrombolytic treatment. Magn Reson Imaging 19: 143-152.

29. Tsai YH, Yuan R, Huang YC, Weng HH, Yeh MY, et al. (2014) Altered resting state FMRI signals in acute stroke patients with ischemic penumbra. PLoS One9: 105117.

30. Zhu S, Fang Z, Hu S, Wang Z, Rao H (2013) Resting state brain function analysis using concurrent BOLD in ASL perfusion fMRI. PLoS One: 65884.

31. Ween JE (2008) Functional imaging of stroke recovery: an ecological review from a neural network perspective with an emphasis on motor systems. J Neuroimaging 18: 227-236

32. Ovadia-Caro S, Margulies DS, Villringer A (2014) The value of resting-state functional magnetic resonance imaging in stroke. Stroke 45: 2818-2824.

33. Ovadia-Caro S, Villringer K, Fiebach J, Jungehulsing GJ, Van der Meer E, et al. (2013) Longitudinal effects of lesions on functional networks after stroke. J Cereb Blood Flow Metab 33: 1279-1285. 